\title{
Vitamin D, UV-Strahlung und Hautkrebs
}

\author{
Die interdisziplinäre Bestandesaufnahme der aktuellen Daten ergibt, dass die UV- \\ Exposition kein geeignetes Mittel zur Korrektur von Vitamin-D-Mangelzuständen \\ darstellt. Die orale Substitution ist zuverlässig und sicher und vermeidet UV-indu- \\ zierte Hautschäden.
}

Marjam-Jeanette Barysch ${ }^{a}$, Heike Bischoff-Ferrari ${ }^{b, c}$, Günther Hofbauera, Reinhard Dummera

a Dermatologische Klinik, UniversitätsSpital Zürich

b Zentrum Alter und Mobilität, Universität Zürich

c Rheumatologie und Institut für Physikalische Medizin UniversitätsSpital Zürich

Interessenbindungen: keine

Korrespondenz:

Prof. Dr. med.

Reinhard Dummer

Stv. Klinikdirektor

Dermatologische Klinik,

UniversitätsSpital Zürich

Gloriastrasse 31

CH-8091 Zürich

Tel. 0442552507

Fax 0442558988

reinhard.dummer@usz.ch

\section{Zusammenfassung}

Vitamin-D-Versorgung ist wichtig vor allem zur Prävention von Stürzen und Knochenbrüchen im Alter. Eine optimierte Vitamin-D-Versorgung wirkt sich auch auf andere Organsysteme positiv aus. Vitamin-D (Calciferol) wird in der Haut unter UV-Einwirkung gebildet. Deshalb erhält es in unserer Gesellschaft, in welcher gebräunte Haut häufig als Schönheitsideal gilt, in der Fachwelt und den Medien grosse Aufmerksamkeit. Einige Autoren fordern eine Lockerung der Sonnenprotektion, andere propagieren sogar regelmässige UV-Exposition zur Risikominderung Vitamin D beeinflussbarer Krankheiten.

Allerdings bewirkt ein Teil des UV-Lichtes, der für die Vitamin-D-Produktion verantwortlich ist, direkte kanzerogene Schäden an der DNA. Zudem unterliegt die kutane Vitamin-D-Bildung erheblichen Schwankungen. Vor diesem Hintergrund ergab eine interdisziplinäre Bestandesaufnahme der aktuellen Daten, dass die UV-Exposition kein geeignetes Mittel zur Korrektur von Vitamin-D-Mangelzuständen darstellt. Die orale Substitution ist zuverlässig und sicher und vermeidet UV-induzierte Hautschäden.

\section{Vitamin D}

Vitamin $\mathrm{D}_{3}$ (Cholecalciferol) spielt eine zentrale Rolle in der Calcium- und Phosphat-Aufnahme [1] und übt einen direkten Effekt auf die Muskulatur aus [3-5]. Weitere Wirkungen wie die Prävention von kardiovaskulären, autoimmunen und malignen Erkrankungen werden mit einem 25-HydroxyvitaminD-Spiegel (der sogenannten «Speicherform» von Vitamin D) von über $75 \mathrm{nmol} / \mathrm{l}$ (30 ng/ml) in Verbindung gebracht $[6-9,2]$.

Die empfohlene Tagesmenge von mindestens 800 IU Vitamin D pro Tag bei Erwachsenen kann über die Nahrung alleine nicht ausreichend gedeckt werden [6]. Neben der peroralen und parenteralen Aufnahme wird Vitamin D auch über UV-Einstrahlung durch Photosynthese dem Körper zur Verfügung gestellt. Hierfür werden UVB-Strahlen mit einer Wellenlänge von 280-315 $\mathrm{nm}$ benötigt [10]. Allerdings ist die maximal induzierbare Vitamin-D-Konzentration bereits in suberythematogenen UV-Dosen erreicht; jede weitere

\section{Vitamine D, rayonnement UV et cancer de la peau}

Selon l'état des données actuelles et conformément à la recommandation de différents experts, le taux souhaitable de $25-\mathrm{OH}-\mathrm{D}_{3}$ est de $75-100 \mathrm{nmol} / \mathrm{l}$. L'exposition intentionnelle aux UV ne constitue nullement un moyen approprié à la correction des états de carence en vitamine $\mathrm{D}$ vu un certain nombre de paramètres comme l'effet cancérigène de l'exposition aux UV, qui se superpose à la photosynthèse de la vitamine $D$, les variations saisonnières et journalières de I'intensité de l'ensoleillement ainsi que les capacités de formation de la vitamine $D$, variables selon les sujets. Une correction de la teneur en vitamine $D$ doit être faite par une supplémentation sous forme de gouttes ou de comprimés chez les sujets sains, tandis que chez les sujets présentant une malabsorption, une tentative d'administration par voie orale peut être tentée en première intention complétée, le cas échéant par des injections intramusculaires de vitamine $\mathrm{D}$. Une attention toute particulière doit être apportée aux groupes à risque, les personnes âgées et les obèses. Pour ce qui concerne le soleil, les mesures de protection solaire connues doivent être suivies.

UV-Exposition führt zu einem Abbau von Vitamin D und dessen Vorstufen in der Haut [10]. Die notwendige UV-Exposition zur maximalen Vitamin-D-Photosynthese ist bei dunkelhäutigen Menschen um den Faktor 6 verlängert, da durch den höheren Melaningehalt der Haut vermehrt UV-Strahlen absorbiert werden. Bei älteren Personen nimmt die Photosynthesekapazität 4-fach ab, verglichen mit jüngeren Personen, und auch adipöse Personen weisen eine verminderte Kapazität zur Vitamin-D-Bildung auf [11, 12]. 


\section{UV-Exposition und Karzinogenese}

UV-Strahlung (UV-A und UV-B) ist nachweislich der wichtigste exogene Risikofaktor für Hautkrebserkrankungen [13-16, 25]. Weltweit steigende Raten werden insbesondere auch in der Schweiz, dem Land mit einer der höchsten Hautkrebsraten, registriert [17]. Zur Erhöhung des Vitamin-D-Spiegels fordern einige Autoren die Lockerung der Sonnenprotektion, manche empfehlen sogar die Benutzung von Solarien oder UV-BLampen $[18,19]$. Da für die Photosynthese hauptsächlich UV-B-Strahlung benötigt wird, Solarien aber überwiegend UV-A-Strahlen abgeben, spielen diese bei der Vitamin-D-Bildung, wenn überhaupt, eine untergeordnete Rolle [14], bei erhaltenem Melanomrisiko [20].

Eine sogenannte «sichere Sonnenexposition» [21] ist nicht definierbar, da gleichzeitig mit der VitaminD-Produktion die DNA-Schädigung beginnt und demzufolge die Karzinogenese einsetzt [11, 25]. Die oftmals angeführte Empfehlung der täglichen Exposition von einem Viertel der Körperoberfläche mit einem Viertel der persönlichen minimalen Erythemdosis (MED) [22] basiert auf In-vitro-Tests und kann nicht auf die Situation in vivo übertragen werden. Zudem ist die individuelle Vitamin-D-Synthese abhängig vom Hauttyp, Pigmentgehalt der Haut, Körpergewicht, und diversen Umweltfaktoren. Ein verminderter Vitamin-D-Spiegel in Zusammenhang mit der kontinuierlichen Anwendung von Lichtschutzmassnahmen konnte bei der Normalbevölkerung nicht nachgewiesen werden [23].

\section{Fazit}

Anhand der heutigen Datenlage und entsprechend der Empfehlung verschiedener Experten ist ein $25(\mathrm{OH}) \mathrm{D}_{3-}$ Spiegel von 75-100 nmol/l anzustreben. Angesichts der kanzerogenen Wirkung der UV-Exposition, die mit der Vitamin-D-Photosynthese überlappt, der saisonalen und tagesabhängigen Schwankungen der Sonnenintensität sowie der individuell unterschiedlichen Kapazitäten zur Vitamin-D-Bildung, ist intendierte UV-Exposition kein geeignetes Mittel zur Korrektur von Vitamin-D-Mangelzuständen. Eine Korrektur des Vitamin-D-Haushaltes sollte durch Supplementierung erfolgen: Bei gesunden Personen kann dies in Form von Tropfen oder Tabletten erfolgen, bei Malabsorption kann primär eine orale Supplementation versucht werden und gegebenenfalls durch intramuskuläre Vitamin-D-Injektionen unterstützt werden [6]. Ein besonderes Augenmerk ist auf Risikogruppen, Ältere und Adipöse, zu richten. In der Sonne sollten die bekannten Sonnenschutzmassnahmen befolgt werden [24].

\section{Literatur:}

1 Holick MF. Vitamin D: its role in cancer prevention and treatment. Prog Biophys Mol Biol. 2006; 92(1):49-59.

2 Wolpowitz D, Gilchrest BA. The vitamin D questions: how much do you need and how should you get it? J Am Acad Dermatol. 2006;54(2):301-17.

3 Bischoff-Ferrari HA, et al. Positive association between 25-hydroxy vitamin D levels and bone mineral density: a population-based study of younger and older adults. Am J Med. 2004;116(9):634-9.
4 Bischoff-Ferrari HA, et al. Higher 25-hydroxyvitamin D concentrations are associated with better lower-extremity function in both active and inactive persons aged $>$ or $=60$ y. Am J Clin Nutr. 2004;80(3):752-8.

5 Bischoff-Ferrari HA, et al. Prevention of nonvertebral fractures with oral vitamin $\mathrm{D}$ and dose dependency: a meta-analysis of randomized controlled trials. Arch Intern Med. 2009;169(6):551-61.

6 Bischoff-Ferrari HA, et al. Estimation of optimal serum concentrations of 25-hydroxyvitamin D for multiple health outcomes. Am J Clin Nutr. 2006 84(1):18-28.

7 Cantorna MT. Vitamin D and its role in immunology: multiple sclerosis, and inflammatory bowel disease. Prog Biophys Mol Biol. 2006;92(1):60-4.

8 Dobnig $\mathrm{H}$, et al. Independent association of low serum 25-hydroxyvitamin d and 1,25-dihydroxyvitamin d levels with all-cause and cardiovascular mortality. Arch Intern Med. 2008;168(12):1340-9.

9 Forman JP, et al. Plasma 25-hydroxyvitamin D levels and risk of incident hypertension. Hypertension, 2007; 49(5):1063-9.

10 Holick MF. Environmental factors that influence the cutaneous production of vitamin D. Am J Clin Nutr. 1995; 61(3 Suppl):638S-645S.

11 Gilchrest BA. Sun protection and Vitamin D: three dimensions of obfuscation. J Steroid Biochem Mol Biol. 2007; 103(3-5):655-63.

12 Webb AR. Who, what, where and when-influences on cutaneous vitamin D synthesis. Prog Biophys Mol Biol. 2006; 92(1):17-25.

13 Dummer R, Maier T, UV protection and skin cancer. Recent Results Cancer Res. 2002;160:7-12.

14 MacKie, RM, Long-term health risk to the skin of ultraviolet radiation. Prog Biophys Mol Biol, 2006.92(1):p. 92-6.

15 Dummer R, B.M. Morcinek J, Burg G. Neoplasien der Haut. Praxis. 2003;92(36):1470-1478.

16 Pfeifer GP, You YH, Besaratinia A. Mutations induced by ultraviolet light. Mutat Res. 2005;571(1-2):19-31.

17 Pury P, et al. Statistics of Cancer Incidence 1986-2005. S.A.o.C. Registries. Editor; 2007.

18 Holick MF, et al. Vitamin D and skin physiology: a D-lightful story. J Bone Miner Res. 2007;22 Suppl 2:V28-33.

19 Koutkia P, et al. Treatment of vitamin D deficiency due to Crohn's disease with tanning bed ultraviolet B radiation. Gastroenterology. 2001;121(6):1485-8.

20 IA, T.I.A.f.R.o.C.W.G.o.a.u.U.l.a.s.c. The association of use of sunbeds with cutaneous malignant melanoma and other skin cancers: A systematic review. In J Int Cancer. 2006; 1116-1122.

21 Boucher BJ, Sunlight «D»ilemma. Lancet. 2001;357(9260):961.

22 Webb AR, Kline L, Holick MF. Influence of season and latitude on the cutaneous synthesis of vitamin D3: exposure to winter sunlight in Boston and Edmonton will not promote vitamin D3 synthesis in human skin. J Clin Endocrinol Metab. 1988;67(2):373-8.

23 Marks R, et al. The effect of regular sunscreen use on vitamin D levels in an Australian population. Results of a randomized controlled trial. Arch Dermatol. 1995;131(4):415-21.

24 Lautenschlager S, Wulf HC, Pittelkow MR. Photoprotection. Lancet. 2007;370(9586):528-37.

25 International Agency for Research on Cancer. Special report policy: A review of human carcinogens - Part D: radiation. Lancet. 2009;(10)8. 